Review Article

\title{
Decay of solutions for 2D navier-stokes equations posed on rectangles and on a half-strip
}

\section{Abstract}

Initial-boundary value problems for 2D Navier-Stokes equations posed on rectangles and on a half-strip were considered. The existence and uniqueness of regular global solutions on rectangles and their exponential decay as well as exponential decay of generalized solutions on a half-strip have been established.

Keywords: navier-stokes equations, lipschitz and smooth domains, decay in bounded and unbounded domains
Volume I Issue 5 - 2018

Larkin NA, Padilha MV

Departamento de Matemática, Universidade Estadual de Maringá, Brazil

Correspondence: Larkin NA, Departamento de Matemática, Universidade Estadual de Maringá, Av. Colombo 5790,Agência UEM, 87020-900, Maringá, PR, Brazil, Email nlarkine@uem.br

Received: June 26, 2018 | Published: September 27, 2018

\section{Introduction}

The main goal of this work is establishing of sharp estimates for the exponential decay rates of solutions to initial-boundary value problems for the 2D Navier-Stokes equations:

$$
\begin{aligned}
& u_{t}+(u \cdot \nabla) u=v \Delta u-\nabla p, \text { in } \Omega \times(0, t), \\
& \nabla u=0 \text { in } \Omega,\left.\quad u\right|_{\partial \Omega}=0, \\
& u(x, y, 0)=u_{0}(x, y),
\end{aligned}
$$

where $\Omega$ is either a bounded rectangle or a half-strip in $\mathbb{R}^{2}$ with the homogeneous Dirichlet condition on the boundary of $\Omega$.

The question of decay of the energy for generalized solutions had been stated by J.Leray ${ }^{1}$ and attracts till now attention of many pure and applied mathematicians ${ }^{2-11}$. In all of these papers, the decay rate of $\|u\|(t)_{L^{2}(\Omega)}$ was controlled by the first eigenvalue of the operator $A=-P \Delta$, where $P$ is the projection operator on solenoidal subspace of $L^{2}(\Omega)$. Associated with stability questions, problems on dimensions of attractors and nonlinear spectral manifolds also have been studied. ${ }^{2,6,7}$

It is well-known that solutions of the 2D Navier-Stokes equations posed on smooth bounded domains with the Dirichlet boundary conditions are globally regular., ${ }^{911-14}$ On the other hand, the question of regularity is not obvious in the case of bounded Lipschitz domains and unbounded Lipschitz and smooth domains. It has been proved that for Lipschitz domains, bounded and unbounded, there exists a unique global generalized solution. ${ }^{9,11,14}$

$$
u, u_{t} \in L^{\infty}\left(0, \infty ; L^{2}(\Omega)\right) \cap L^{2}\left(0, \infty ; H^{1}(\Omega)\right),
$$

but it was not clear whether

$$
u \in L^{\infty}\left(0, \infty ; H^{2}(\Omega)\right)
$$

at least for bounded Lipschitz domains.

In this work, we have established this fact for rectangles making use of ideas. ${ }^{15}$ The following inequality holds for rectangles

$$
\|u\|_{H^{2}(\Omega)}^{2}(t)+\left\|u_{t}\right\|_{L^{2}(\Omega)}^{2}(t) \leq C\left\|u_{0}\right\|_{H^{2}(\Omega)}^{2} \exp \left(-v\left(\frac{\pi^{2}}{L^{2}}+\frac{\pi^{2}}{B^{2}}\right) t\right)
$$

and

$$
\|u\|_{H^{1}(\Omega)}^{2}(t)+\left\|u_{t}\right\|_{L^{2}(\Omega)}^{2}(t) \leq C\left\|u_{0}\right\|_{H^{2}(\Omega)}^{2} \exp \left(-\frac{v \pi^{2}}{B^{2}} t\right)
$$

for a half-strip.

Our paper has the following structure: Chapter I is Introduction. Chapter 2 contains notations and auxiliary facts. In Chapter 3, existence and uniqueness of global generalized solutions on either bounded or unbounded Lipschitz domains have been established. In Chapter 4, regularity and decay of solutions on rectangles and on a half-strip have been studied.

\section{Notations and auxiliary facts}

Let $\Omega$ be a domain in $\mathbb{R}^{2}$. Define as in:"11

$$
D_{x}=\frac{\partial}{\partial x}, D_{y}=\frac{\partial}{\partial y}, D^{j}=D_{x}^{j_{x}} D_{y}^{j_{y}}=\frac{\partial^{\left|j_{x}+j_{y}\right|}}{\partial x^{j_{x}} \partial y^{j_{y}}} .
$$

We denote for scalar functions $f(x, y, t)$ by $L^{p}(\Omega), 1<p<+\infty$ the Banach space with the norm

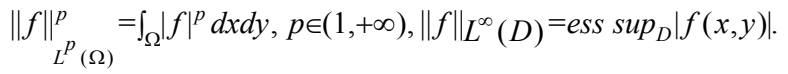

For $p=2, L^{2}(\Omega$ is a Hilbert space with the scalar product

$$
(u, v)=\int_{\Omega} u(x, y) v(x, y) d x d y \text { and the norm }\|u\|^{2}=\int_{\Omega}|u(x, y)|^{2} d x d y .
$$

The Sobolev space $W^{m, p}(\Omega)$ is a Banach space with the norm

$$
\|u\|_{W^{k, p}(\Omega)}=\sum_{0 \leq|\alpha| \leq k}\left\|D^{\alpha} u\right\|_{L^{p}(\Omega)} .
$$

When $p=2, W^{m, 2}(\Omega)=H^{m}(\Omega)$ is a Hilbert space with the following scalar product and the norm:

$$
((u, v))_{H^{m}{ }_{(\Omega)}}=\sum_{|j| \leq m}\left(D^{j} u, D^{j} v\right),\|u\|_{H^{m}(\Omega)}^{2}=\sum_{|j| \leq m}\left\|D^{j} u\right\|^{2} .
$$


Let $\mathcal{D}(\Omega)$ or $\mathcal{D}(\bar{\Omega})$ be the space of $C^{\infty}$ functions with compact support in $\Omega$ or $\bar{\Omega}$. The closure of $C^{\infty}$ functions in $W^{m, p}(\Omega)$ is denoted by $W_{0}^{m, p}(\Omega)$ and $\left(H_{0}^{m}(\Omega)\right.$ when $\left.p=2\right)$.

Define the auxiliary spaces which are projections for the solenoidal vector functions,

$$
\begin{aligned}
& \mathcal{V}=\{u \in \mathcal{D}(\Omega), \nabla u=0\}, V=\text { the closure of } \mathcal{V} \text { in } H_{0}^{1}(\Omega), \\
& H=\text { the closure of } \mathcal{V} \text { in } L^{2}(\Omega),
\end{aligned}
$$

The space $H$ is eqquiped with the natural $L^{2}$ inner product. The space $V$ will be equipped with the scalar produt

$$
((u, v))=\left(D_{x} u, D_{x} v\right)+\left(D_{y} u, D_{y} v\right)
$$

when $\Omega$ is bounded. If $\Omega$ is unbounded, we define the inner product as the sum of the inner products as following:

$$
[[u, v]]=(u, v)+((u, v)) .
$$

We use the usual notations of Sobolev spaces $W^{k, p}, L^{p}$ and $H^{k}$ for vector functions and the following notations for the norms:

i) For vector functions $u(x, y, t)=\left(u_{1}(x, y, t), u_{2}(x, y, t)\right)$,

$$
\begin{gathered}
\|u\|_{L^{p}(\Omega)}^{p}=\int_{\Omega}\left(\left|u_{1}\right|^{p}+\left|u_{2}\right|^{p}\right) d x d y, \\
\|u\|_{W^{k, p}(\Omega)}=\sum_{0 \leq|\alpha| \leq k}\left\|D^{\alpha} u_{1}\right\|_{L^{p}(\Omega)}+\left\|D^{\alpha} u_{2}\right\| L^{p}(\Omega), p \in(1,+\infty) .
\end{gathered}
$$

The closures of $\mathcal{V}$ in $L^{2}(\Omega)$ and in $H_{0}^{1}(\Omega)$ are the basic spaces in our study. We denote them by $H$ and $V$ respectively.. Obviously $V$ is a subspace of $H_{0}^{1}(\Omega)$.

Define the operator

$$
(u \cdot \nabla) u=\left(u_{1} u_{1 x}+u_{1} u_{2 x}+u_{2} u_{1 y}+u_{2} u_{2 y}\right) .
$$

Lemma 4.1 (The Steklov Inequality) ${ }^{16}$ Let $v \in H_{0}^{1}(0, L)$. Then

$$
\frac{\pi^{2}}{L^{2}}\|v\|^{2}(t) \leq\left\|v_{x}\right\|^{2}(t) .
$$

Proof. Let $v(t) \in H_{0}^{1}(0, \pi)$, then by the Fourier series,

$$
\int_{0}^{\pi} v_{t}^{2}(t) d t \geq \int_{0}^{\pi} v^{2}(t) d t
$$

Inequality (4) follows by a simple scaling.

Lemma 4.2 (Differential form of the Grownwall Inequality) Let $I=\left[t_{0}, t_{1}\right]$. Suppose that functions $a, b: I \rightarrow \mathbb{R}$ are integrable and a function $a(t)$ may be of any sign. Let $u: I \rightarrow \mathbb{R}$ be a differentiable function satisfying

$$
u^{\prime}(t) \leq a(t) u(t)+b(t), \text { for } t \in \operatorname{Iand} u\left(t_{0}\right)=u_{0},
$$

then

$$
u(t) \leq u_{0} e^{\int_{0}^{t} a(t) d t}+\int_{t_{0}}^{t} e^{\int^{s} a(r) d r} b(s) d s
$$

Proof. Multiply (5) by the integrating factor $e^{\int_{t_{0}}^{S} a(r) d r}$ and integrate from $t_{0}$ to $t$.

The next Lemmas will be used in estimates:

Lemma 4.3 (See: ${ }^{11,14}$ ) Let $v \in H_{0}^{1}(\Omega)$, then

$$
\|v\|_{L^{4}(\Omega)} \leq 2^{1 / 4}\|v\|_{L^{2}(\Omega)}^{1 / 2}\|\nabla v\|_{L^{2}(\Omega)}^{1 / 2} .
$$

Lemma $4.4\left(\right.$ See: $\left.{ }^{14}\right)$ Let $b(u, v, w)=((u \cdot \nabla) v, w)$, then

$$
|b(u, v, w)| \leq 2^{1 / 2}\|u\|^{1 / 2}\|u\|_{V}^{1 / 2}\|v\|_{V}\|w\|^{1 / 2}\|w\|_{V}^{1 / 2}
$$

$\forall u, v, w \in H_{0}^{1}(\Omega)$. If $u \in L^{2}(0, \infty ; V) \cap L^{\infty}(0, \infty ; H)$, then we can define the operator $B u$ such that $B u$ belongs to $L^{2}\left(0, \infty ; V^{\prime}\right)$ and

$$
\begin{aligned}
& (B u, v)=b(u, u, v), \\
& \left\|B_{u}\right\|_{L^{2}\left(0, \infty ; V^{\prime}\right)} \leq 2^{1 / 2}\|u\|_{L^{\infty}(0, \infty ; H)}\|u\|_{L^{2}(0, \infty ; V)} .
\end{aligned}
$$

\section{Existence theorems}

Let $\Omega$ be a bounded Lipschitz domain. Given $u_{0} \in H$, consider the following problem:

$$
\left\{\begin{array}{c}
u_{t}-v \Delta u+\nabla p+(u \cdot \nabla) u=0 \operatorname{in} \Omega \times(0, t), \\
\nabla u=0 \operatorname{in} \Omega \times(0, t), \\
u=0 \text { on } \partial \Omega \times(0, t), t>0, \\
u(x, y, 0)=u_{0}(x, y), \operatorname{in} \Omega
\end{array}\right.
$$

equivalent to the variational problem given by, ${ }^{11}$

$$
\left\{\begin{array}{c}
u^{\prime}+A u+B u=0 \text { in }(0, t), t>0 \\
u(0)=u_{0},
\end{array}\right.
$$

where $A u \in V^{\prime}$ such that $(A u, v)=-v((u, v))$ for all $v \in V$ and $B u \in V^{\prime}$ such that

$$
(B u, v)=b(u, u, v) .
$$

Theorem 5.1 Given $u_{0} \in H^{2}(\Omega) \cap V$, there exists a unique generalized solution $u$ to (8) such that for all $\Phi \in V,\left.\Phi\right|_{\partial \Omega}=0$ it satisfies the following integral identity:

$$
\int_{\Omega}\left\{u_{t} \Phi+v\left(u_{x} \Phi_{x}+u_{y} \Phi_{y}\right)-u(u \cdot \nabla) \Phi\right\} d x d y=0,
$$

where

$$
u \in L^{\infty}(0, \infty ; V), u_{t} \in L^{\infty}(0, \infty ; H) \cap L^{2}(0 ; \infty ; V) .
$$

Proof. The estimates that follow may be established on Gallerkin approximations. ${ }^{14,9}$ We estimate:

Estimate I - $u \in L^{\infty}(0, \infty ; H) \cap L^{2}(0, \infty ; V)$.

Multiply (9) by $u$ to obtain

$$
\left(u_{t}, u\right)(t)+(A u, u)(t)=0 .
$$

It follows from here that

$$
\frac{d}{d t}\|u\|^{2}(t)+2 v\|u\|^{2}(t)_{V}=0
$$


Integrating $(13)$ over $(0, t)$, we get

$\|u\|^{2}(t)+2 v \int_{0}^{t}\|u\|_{V}^{2}(s) d s=\left\|u_{0}\right\|^{2}, t>0$.

Hence $u \in L^{\infty}(0, \infty ; H) \cap L^{2}(0, \infty ; V)$.

Estimate II - $u_{t} \in L^{\infty}(0, \infty ; H) \cap L^{2}(0, \infty ; V)$.

Derivating (9) and multiplying by $u_{t}$, we get

$\frac{d}{d t}\left\|u_{t}\right\|^{2}(t)+2 v\left\|u_{t}\right\|_{V}^{2}(t)+2 b\left(u_{t}, u, u_{t}\right)(t)=0$.

By Lemma 4.4,

$$
\begin{aligned}
& 2\left|b\left(u_{t}, u, u_{t}\right)(t)\right| \leq 2^{3 / 2}\left\|u_{t}\right\|(t)\left\|u_{t}\right\|_{V}\|u\|_{V} \\
& \leq v\left\|u_{t}\right\|_{V}^{2}+\frac{2^{3}}{v}\|u\|_{V}^{2}(t)\left\|u_{t}\right\|^{2}(t)
\end{aligned}
$$

and (15) becomes

$$
\frac{d}{d t}\left\|u_{t}\right\|^{2}(t)+v\left\|u_{t}\right\|_{V}^{2}(t) \leq \phi(t)\left\|u_{t}\right\|^{2}(t),
$$

where $\phi(t)=\frac{2^{3}}{v}\|u\|_{V}^{2}(t)$. Making use of Lemma 4.2, we obtain

$$
\left\|u_{t}\right\|^{2}(t) \leq\left\|u_{t}\right\|^{2}(0) e^{\int_{0}^{t} \phi(s) d s} .
$$

To prove that $\left\|u_{t}\right\|(0)$ is in $H$, multiply equation (9) by $u_{t}(t)$ to get

$$
\left\|u_{t}\right\|^{2}(t)+v\left(\left(u, u_{t}\right)\right)(t)+b\left(u, u, u_{t}\right)(t)=0 .
$$

In particular, for $t=0$ we have

$\left\|u_{t}(0)\right\|^{2}=v\left(\Delta u_{0}, u_{t}(0)\right)-b\left(u_{0}, u_{0}, u_{t}(0)\right)$,

where $u_{t}(0)=\lim _{t \rightarrow 0} u_{t}(t)$, [19]. From this

$$
\left\|u_{t}(0)\right\| \leq v\left\|\Delta u_{0}\right\|+\left\|B u_{0}\right\| .
$$

By the Hölder inequality,

$$
\begin{aligned}
& |b(u, u, v)| \leq\|u\|_{L^{4}(\Omega)}\left(2\|\nabla u\|_{L^{4}(\Omega)}+\left\|u_{2 x}\right\|_{L^{4}(\Omega)}+\left\|u_{1 y}\right\|_{L^{4}(\Omega)}\right)\|v\| \\
& \leq C\|u\|_{V}\|u\|_{H^{2}(\Omega)}\|v\|, \quad \forall u \in H^{2}(\Omega), \forall v \in L^{2}(\Omega) .
\end{aligned}
$$

Hence

$$
\left|B u_{0}\right| \leq C|| u_{0}\left\|_{V}\right\| u_{0}\left\|_{H^{2}(\Omega)} \leq C|| u_{0}\right\|_{H^{2}(\Omega)}^{2}
$$

and by (18), $u_{t}(0) \in H$. This and (16) imply that

$$
u_{t} \in L^{\infty}(0, \infty ; H) \cap L^{2}(0, \infty ; V) .
$$

Returning to (12), we calculate

$$
v\|u\|_{V}^{2}(t)=\left(u, u_{t}\right)(t) \leq\|u\|(t)\left\|u_{t}\right\|(t),
$$

hence $u \in L^{\infty}(0, \infty ; V)$. This and (17) prove validity of (11) and consequently the existence part of Theorem 3.1. Uniqueness of the generalized solution, $u, u_{t} \in L^{\infty}(0, \infty ; H) \cap L^{2}(0, \infty ; V)$ has been established. ${ }^{9,14}$
Remark 5.1 Estimates $u, u_{t} \in L^{\infty}\left(0, \infty ; H \cap L^{2}(0, \infty ; V)\right.$ were established first for Lipschitz domains ${ }^{9,14}$ and were valid also for unbounded domains with a natural condition $\lim _{|x| \rightarrow+\infty} u(x, y, t)=0$. We repeat them because we will need these estimates while establishing decay of solutions in bounded and unbounded Lipschitz domains.

\section{Regularity and decay on rectangles and on the half-strip}

Consider the Poisson problem in a bounded rectangle $\Omega$

$$
\left\{\begin{array}{c}
\Delta u=f(x, y),(x, y) \in \Omega, \\
\left.u\right|_{\partial \Omega}=0,
\end{array}\right.
$$

Remark 6.1 It has been proved ${ }^{10}$ that for

$$
\Omega_{\pi}=\left\{x=\left(x_{1}, \ldots, x_{n}\right), \quad 0<x_{i}<\pi ; i=1, \ldots, n\right\}
$$

the following inequality holds

$$
\|u\|_{W}^{2, p}\left(\Omega_{\pi}\right) \leq C(\Omega)\|f\|_{L}{ }_{\left(\Omega_{\pi}\right)} .
$$

It is easy to generalize this result for any rectangle in $\mathbb{R}^{2}$.

Theorem 6.1 The problem (23) posed in rectangle $\Omega=\left\{(x, y) \in \mathbb{R}^{2}, 0<x<L ; 0<y<B\right\}$, where $f \in L^{p}(\Omega), \quad 1<p \leq 2$, has a solution $u \in W^{2, p}(\Omega)$. Moreover,

$$
\|u\|_{W^{2, p}}{ }_{(\Omega)} \leq c_{\Omega}\|f\|_{L}^{p}(\Omega) .
$$

Returning to the original problem for the Navier-Stokes equations,

$$
\left\{\begin{array}{c}
u_{t}-v \Delta u+\nabla p+(u \cdot \nabla) u=0 \operatorname{in} \Omega \times(0, t) \\
\nabla u=0 \operatorname{in} \Omega \times(0, t), \\
u=0 \operatorname{in} \partial \Omega \times(0, t), t>0, \\
u(x, y, 0)=u_{0}(x, y), \operatorname{in} \Omega,
\end{array}\right.
$$

where $u$ is a vector function from $\mathbb{R}^{2}$ into $\mathbb{R}^{2}$ and $p$ is a real function from $\mathbb{R}^{2}$ into $\mathbb{R}$, and making use of Galerkin approximations, we establish the following result.

Theorem 6.2 Given $u_{0} \in H^{2}(\Omega) \cap V$, the problem (25) has a unique solution $(u, p)$ such that

$$
\begin{aligned}
& u \in L^{\infty}\left(0, \infty ; V \cap H^{2}(\Omega)\right), u_{t} \in L^{\infty}(0, \infty ; H) \cap L^{2}(0, \infty ; V), \\
& \nabla p \in L^{\infty}(0, \infty ; H) .
\end{aligned}
$$

Moreover,

$$
\left\|u_{t}\right\|(t)+\|u\|(t)_{H^{2}(\Omega)}+\|\nabla p\|(t) \leq C e^{-\frac{1}{2} \chi t},
$$

where $\chi=v\left(\frac{\pi^{2}}{L^{2}}+\frac{\pi^{2}}{B^{2}}\right)$ and $C$ depends on $\left\|u_{0}\right\|_{H^{2}(\Omega)}$.

\section{Proof. Decay of $L^{2}$ Norm}

By definition,

$$
\|u\|_{V}^{2}(t)=\left\|u_{x}\right\|^{2}(t)+\left\|u_{y}\right\|^{2}(t) .
$$

Since $\left.u\right|_{\partial \Omega}=0$, making use of Lemma 4.1, we get 
$\left\|u_{x}\right\|^{2}(t) \geq \frac{\pi^{2}}{L^{2}}\|u\|^{2}(t), \quad\left\|u_{y}\right\|^{2}(t) \geq \frac{\pi^{2}}{B^{2}}\|u\|^{2}(t)$

This implies $\|u\|_{V}^{2}(t) \geq\left(\frac{\pi^{2}}{L^{2}}+\frac{\pi^{2}}{B^{2}}\right)\|u\|^{2}(t)$.

Returning to (12), we obtain

$$
\frac{d}{d t}\|u\|^{2}(t)+2 v\left(\frac{\pi^{2}}{L^{2}}+\frac{\pi^{2}}{B^{2}}\right)\|u\|^{2}(t) \leq 0 .
$$

Define $\chi=v\left(\frac{\pi^{2}}{L^{2}}+\frac{\pi^{2}}{B^{2}}\right)$. Then (29) implies

$$
\|u\|^{2}(t) \leq\left\|u_{0}\right\|^{2} e^{-2 \chi t} .
$$

\section{Decay of $H^{1}$ Norm}

Rewrite (15) in the form

$$
\frac{d}{d t}\left\|u_{t}\right\|^{2}(t)+v\left\|u_{t}\right\|_{V}^{2}(t)-\phi(t)\left\|u_{t}\right\|^{2}(t) \leq 0
$$

where $\phi=\frac{2^{3}}{v}\|u\|_{V}^{2}(t)$. Acting similarly to the proof of (29), we obtain

$$
\left\|u_{t}\right\|_{V}^{2}(t) \geq\left(\frac{\pi^{2}}{L^{2}}+\frac{\pi^{2}}{B^{2}}\right)\left\|u_{t}\right\|^{2}(t) .
$$

Hence (31) reduces to the form

$$
\frac{d}{d t}\left\|u_{t}\right\|^{2}(t)+(\chi-\phi(t))\left\|u_{t}\right\|^{2}(t) \leq 0
$$

By Lemma 4.2,

$$
\left\|u_{t}\right\|^{2}(t) \leq\left\|u_{t}(0)\right\|^{2} e^{\int_{0}^{t} \phi(s) d s} e^{-\chi t} .
$$

Since $u \in L^{2}(0, \infty, V)$, then by (14),

$$
\int_{0}^{t} \phi(s) d s \leq \frac{2}{v}\left\|u_{0}\right\|^{2}, t>0,
$$

and it follows from (13) that

$$
\begin{aligned}
& v\|u\|_{V}^{2}(t) \leq\left(u, u_{t}\right)(t) \leq\|u\|(t)\left\|u_{t}\right\|(t) \\
& \leq\left\|u_{0}\right\|\left\|u_{0^{\prime}}\right\| e_{v}^{-\frac{2}{-\left\|u_{0}\right\|}} e^{-\chi t} e^{-\frac{1}{2} \chi t} .
\end{aligned}
$$

Therefore $\|u\|_{V}^{2}(t) \leq \frac{1}{v}\left\|u_{0}\right\|\left\|u_{0^{\prime}}\right\| e_{V}^{-}\left\|u_{0}\right\| e^{-\frac{3}{2} \chi t}$

$$
\text { and }\|u\|_{H_{0}^{1}(\Omega)}^{2}(t) \leq\left(\frac{1}{v}\left\|u_{0}\right\|\left\|u_{0^{\prime}}\right\| e_{v}^{-\frac{2}{-\left\|u_{0}\right\|}}+\left\|u_{0}\right\|^{2}\right) e^{-\frac{3}{2} \chi t}
$$

\section{Decay of $H^{2}$-Norm}

In order to estimate $\|u\|_{H^{2}(\Omega)}(t)$, we will use Theorem 6.1. First write (8) as

$$
\Delta u=f=\frac{1}{v}\left(u_{t}+\nabla p-(u \cdot \nabla) u\right) .
$$

We estimate

$|b(u, u, v)|(t)=|((u \cdot \nabla), v)(t)| \leq c_{2}\|u\|(t)_{L^{4}(\Omega)}\|u\|(t)_{H_{0}^{1}(\Omega)}\|v\|(t)_{L^{4}(\Omega)}$

$\leq C\|u\|^{2}(t)_{H_{0}(\Omega)}^{1}\|v\|(t)_{L}^{4}(\Omega)$

and by (30),

$\|(u \cdot \nabla) u\|(t)_{L}^{4 / 3}(\Omega) \leq C e^{-\frac{3}{2} \chi t}$.

Returning to (9), we obtain

$\|A u\|_{L^{4 / 3}(\Omega)}(t) \leq\|B u\|_{L^{4 / 3}(\Omega)}(t)+\left\|u_{t}\right\|_{L^{2}(\Omega)}(t)$.

It follows by (38) and (34) that $\|A u\|(t)_{L}{ }^{4 / 3} \leq C e^{-\frac{1}{2} \chi t}$. By Theorem of de Rham, ${ }^{17}$ one can check that there exists $\nabla p$ such that ${ }^{11}$

$$
-\nabla p=u_{t}+A u+B u
$$

and

$$
\begin{aligned}
& \|\nabla p\|_{L^{4 / 3}(\Omega)}(t) \leq\left\|u_{t}\right\|_{L^{2}(\Omega)}(t)+\|A u\|_{L^{4 / 3}(\Omega)}^{4 / 3}(t) \\
& +\|B u\|_{L^{4 / 3}(\Omega)}(t) \leq C e^{-\frac{1}{2} \chi t} .
\end{aligned}
$$

Since $f \in L^{4 / 3}(\Omega)$, due to Theorem 6.1,

$$
\begin{aligned}
& \|u\|(t)_{W} 2, \frac{4}{3(\Omega)} \leq\left\|u_{t}\right\|_{L^{2}(\Omega)}(t)+\|\nabla p\|_{L^{4 / 3}(\Omega)}(t) \\
& +\|(u \cdot \nabla) u\|_{L^{4 / 3}(\Omega)}(t)
\end{aligned}
$$

and by (42), we get $\|u\|(t)_{W}^{2,4 / 3}(\Omega) \leq C e^{-\frac{1}{2} \chi t}$. By the Sobolev theorems,

$$
\|u\|_{L^{\infty}(\Omega)}(t) \leq C\|u\|_{W{ }_{(\Omega)}^{2,4 / 3}}(t) \leq C e^{-\frac{1}{2} \chi t} .
$$

This implies

$$
\|B u\|(t) \leq C\|u\|(t)_{L}^{\infty}(\Omega)\|u\|(t)_{H_{0}^{1}(\Omega)} \in L^{2}(\Omega) .
$$

To prove that the norms $\left\|u_{t}\right\|_{L^{2}},\|\nabla p\|_{L^{2}(\Omega)}$ and $\|(u \cdot \nabla) u\|_{L^{2}(\Omega)}$ have exponential decay, we use the equality (10)

$$
\|(u \cdot \nabla) u\|(t)=\|B u\|(t),
$$

where $B u \in L^{2}(\Omega)^{\prime}$ such that

$$
\langle B u, v\rangle=\int_{\Omega}\left(u_{1} u_{1 x} v_{1}+u_{1} u_{1 y} v_{2}+u_{2} u_{2 x} v_{1}+u_{2} u_{2 y} v_{2}\right) d \Omega
$$

for every $v \in L^{2}(\Omega)$. We calculate

$$
|b(u, u, v)|(t) \leq C\|u\|_{L^{\infty}(\Omega)}(t)\|u\|_{H_{0}^{1}(\Omega)}(t)\|v\|(t) .
$$

Since the right-hand side of (44) has exponential decay for every $v \in L^{2}(\Omega)$, it follows 


$$
\|(u \cdot \nabla) u\|(t) \leq C e^{-\frac{5}{4} \chi t} .
$$

Returning to (9), we obtain the decay rate for the operator $A u$

$\|A u\|(t) \leq\|B u\|(t)+\left\|u_{t}\right\|(t)$

It follows from (34) and (45) that $\|A u\|(t) \leq C e^{-\frac{1}{2} \chi t}$. By (40),

$$
\|\nabla p\|_{L^{2}}(t) \leq\left\|u_{t}\right\|(t)+\|A u\|(t)+\|B u\|(t) \leq C e^{-\frac{1}{2} \chi t}
$$

Since now $f \in L^{2}(\Omega)$, substituting (34), (45), (46) into (24) and making use of Theorem 6,1 , we prove

$\|u\|_{H^{2}(\Omega)}(t) \leq C e^{-\frac{1}{2} \chi t}$. It means that a unique generalized soliution is regular.

The proof of Theorem 6.2 is complete.

\section{Existence and decay on the half-strip}

Theorem 7.1 Consider the half-strip $\Omega=\left\{(x, y) \in \mathbb{R}^{2} ; 0<x, 0<y<B\right\}$. Given $u_{0} \in H^{2}(\Omega) \cap V$, the following problem:

$$
\left\{\begin{array}{c}
u_{t}-v \Delta u+\nabla p+(u \cdot \nabla) u=0 \operatorname{in} \Omega \times(0, t), \\
\nabla u=0 \operatorname{in} \Omega \times(0, t), \\
u=0 \operatorname{on} \partial \Omega \times(0, t), t>0, \\
\lim _{x \rightarrow \infty}|u(x, y, t)|=0, t>0, \\
u(x, y, 0)=u_{0}(x), \operatorname{in} \Omega
\end{array}\right.
$$

has a unique solution $(u, p)$ such that

$$
\begin{aligned}
& u \in L^{\infty}\left(0, \infty ; H_{0}^{1}(\Omega)\right), u_{t} \in L^{\infty}\left(0, \infty ; L^{2}(\Omega)\right), \\
& \nabla p \in L^{\infty}\left(0, \infty ; L^{2}(\Omega)\right) .
\end{aligned}
$$

Moreover,

$$
\left\|u_{t}\right\|(t)+\|u\|_{H_{0}^{1}(\Omega)}(t)+\|\nabla p\|_{L^{4 / 3}(\Omega)}(t) \leq C_{2} e^{-\frac{1}{2} \theta t},
$$

where $\theta=v \frac{\pi^{2}}{B^{2}}$ and $C_{2}$ depends on $v,\left\|u_{0}\right\|_{H^{2}(\Omega)}$.

Proof. Obviously, the variational formulation of (47) is also (9). Repeating the proof of Theorem 5.1 (see Remark 3.1), we can proof the existence and uniqueness of the generalized solution ${ }^{18}$ to problem (47). Note that (14) holds for the problem (47). Using the Steklov inequality with respect to variable $y$, we obtain

$$
\left\|u_{y}\right\|^{2} \geq \frac{\pi^{2}}{B^{2}}\|u\|^{2}
$$

hence, similarly to (13),

$$
\frac{d}{d t}\|u\|^{2}(t)+2 v \frac{\pi^{2}}{B^{2}}\|u\|^{2}(t)+2 v\left\|u_{x}\right\|^{2}(t) \leq 0 .
$$

By Lemma $5,\|u\|^{2}(t) \leq\left\|u_{0}\right\|^{2} e^{-2 v \frac{\pi^{2}}{B^{2}} t}$.

Since (31) holds for the problem (47), making use of Lemma 4.4, we estimate

$$
\frac{d}{d t}\left\|u_{t}\right\|^{2}(t)+2 v\left\|u_{t}\right\|^{2}(t)_{V} \leq 2\left\|u_{t}\right\|(t)\|u\|(t)_{V}\left\|u_{t}\right\|(t)_{V}
$$

which we rewrite as

$$
\frac{d}{d t}\left\|u_{t}\right\|^{2}(t)+v\left\|u_{t}\right\|_{V}^{2}(t)-\frac{2}{v}\|u\|_{V}^{2}(t)\left\|u_{t}\right\|^{2}(t) \leq 0 .
$$

By Lemma 4.1,

$$
\left\|u_{t y}\right\|^{2}(t) \geq \frac{\pi^{2}}{B^{2}}\left\|u_{t}\right\|^{2}(t)
$$

and (53) becomes

$$
\frac{d}{d t}\left\|u_{t}\right\|^{2}(t)+\left[v \frac{\pi^{2}}{B^{2}}-\frac{2}{v}\|u\|_{V}^{2}(t)\right]\left\|u_{t}\right\|^{2}(t) \leq 0 .
$$

By Lemma 5, (54) provides

$\left\|u_{t}\right\|^{2}(t) \leq\left\|u_{t}\right\|^{2}(0) e^{\frac{2^{3}}{v} \int_{0}^{t}\|u\|_{V}^{2}(s) d s} e^{-v \frac{\pi^{2}}{B^{2}} t}$,

hence

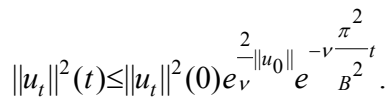

Returning to (35), we estimate

$\|u\|_{V}^{2}(t) \leq \frac{1}{v}\left\|u_{t}\right\|(t)\|u\|(t) \leq \frac{1}{v}\left\|u_{0}\right\|\left\|u_{t}\right\|(0) e^{\frac{2}{v}\left\|u_{0}\right\|} e^{-v \frac{3 \pi^{2}}{2 B^{2}} t}$.

\section{Decay for Pressure}

In order to obtain decay for $\|\nabla p\|_{L}^{4 / 3}{ }_{(\Omega)}(t)$, we start with

$\|(u \cdot \nabla) u\|_{L^{4 / 3}(\Omega)}(t)=\|B u\|_{L^{4}(\Omega)^{\prime}}(t)$,

where $L^{4}(\Omega)^{\prime}$ is the dual of the space $L^{4}(\Omega)$. Since

$A u=-u_{t}-B u$,

repeating calculations of (38) and making use of (34), we get

$\|A u\|(t)_{L}^{4 / 3}(\Omega) \leq c_{1} e^{-\frac{1}{2} \theta t}$. Observing that (40) holds for the problem (47), we obtain

$$
\begin{gathered}
\|\nabla p\|_{L^{4 / 3}\left({ }^{4}\right)}(t) \leq\left\|u_{t}\right\|_{L^{2}(\Omega)}(t)+\|A u\|_{L^{4}(\Omega)}(t) \\
+\|B u\|_{L^{4 / 3}(\Omega)}(t) \leq c_{2} e^{-\frac{1}{2} \theta t} .
\end{gathered}
$$

Jointly (55), (56) and (57) prove (48), (49). 


\section{Conclusion}

In our work, we tried to respond some questions posed by J. Leray, ${ }^{1}$ namely, regularity of global solutions of the Navier-Stokes equations and their decay. Therefore, our results can be divided in two parts: the first one concerns decay of global regular solutions of the 2D Navier-Stokes equations posed on rectangles. ${ }^{19}$ It is known that there exist global regular solutions for the 2D Navier-Stokes equations posed on smooth bounded domains, ${ }^{4,10,11,14}$ but regularity in nonsmooth (Lipschitz) domains, such as rectangles, is not obvious. For bounded rectangles, we have established the existence of an unique global regular solution which decays exponentially as $t \rightarrow+\infty$. We demonstrated that the decay rate is different for different norms, see (26), (30), (36), where $\chi$ is defined by the geometrical characteristics of a domain $\Omega$.

The second part of our work concerns decay of solutions for the 2D Navier-Stokes equations posed on a half-strip. In existing publications, ${ }^{3-11}$ the decay rate of $\|u\|_{L^{2}(\Omega)}(t)$ is controlled by the first eigenvalue of the operator $A=-P \Delta$, where $P$ is the projection operator on solenoidal subspace of $L^{2}(\Omega)$. It is clear that this approach does not work in unbounded domains $\Omega$. On the other hand, our approach based on the Steklov inequality with respect to $y$, allowed us to estimate the decay rate of a generalized solution for the 2D NavierStokes equations posed on a half-strip.

We must emphasize that this estimate is the first in the history which gives an explicit value of the decay rate for unbounded domains. Results established in our work can be used in constructing of numerical schemes for solving initial-boundary value problems for the Navier-Stokes equations appearing in Mechanics of viscous liquid. From the physical point of view, decay estimates show that the decay rate of perturbations of solutions caused by the initial data is bigger for bigger values of viscosity $v$ and smaller values of the width and length of the rectangles and the width of a half-strip.

\section{Acknowledgements}

None.

\section{Conflict of interest}

The authors declare that there are no conflict of interest regarding the publication of this paper.

\section{References}

1. Leray Essai J. Sur le mouvement d'un uide visqueux emplissant l'espace. Acta Math. 1934;63:193-248.

2. Brown RM, Perry P, Shen Z. On the dimension of the attractor for the non-homogeneous Navier-Stokes equations in non-smooth domains. Indiana Univ Math Journal. 2000;40(1):81-112.
3. Edmunds DE. Asymptotic behavior of solutions of the Navier-Stokes equations. Arch Rational Mech Anal. 1966;22(1):15-21.

4. Foias C, Prodi G. Sur le comportement global des solutions nonstationairos des equations de Navier-Stokes en dimension 2. Rendiconti del Seminario Matemtico della Universit di Padova. 1967;39:1-34.

5. Foias C, Saut JC. Asynptotic behavior as t! 1of solutions of Navier-stokes equations in nonlinear partial dierential equations and their applications. College of France Seminar. 1983;4:74-86.

6. Foias C, Temam R. Some analytic and geometric properties of the solutions of the evolution of Navier-Stokes equations. J Math Pures Appl. 1979;58:339-368

7. Foias C, Saut JC. Asymptotic Behavior, as t! 1 of Solutions of NavierStokes Equations and Nonlinear Spectral Manifolds Indiana University Mathematics Journal. 1984;33.10.1512/iumj.1984.33.33025.

8. Guillop C. Comportement l'in ni des solutions des equations de NavierStokes et proprit des ensembles fonctionnels invariants (ou attracteurs). Annales de l'institut Fourier. 1982;32(3):1-37.

9. Ladyzhenskaya O. Solution in the large of the nonstationary boundary value problem for the navier-stokes system with two space variables. Comm Pur Applied Math. 1959;12(3):427-433.

10. Prodi G, Qualche risultato riguardo alle equazioni di Navier-Stokes nel caso bidimensional. Rend Sem Mat Univ Padova. 1965;30:1-15.

11. Temam R. Navier-Stokes Equations. Theory and Numerical Analysis. Noth-Holland, Amsterdam; 1979.

12. Cattabriga L. Su un problema al contorno relativo al sistema di equazioni di Stokes. Rendiconti del Seminario Matematico della Universit di Padova. 1961;31:308-340.

13. Constantin P, Foias C. Navier-Stokes Equations. Chicago Lectures in Mathematics; 1988.

14. Ladyzhenskaya OA. The Mathematical Theory of Viscous Incompressible Flow. 2nd ed. English translation, New York: Gordon and Breach; 1969.

15. Koshelev AI. A priori estimates in Lp and generalized solutions of elliptic equations and systems. Amer Math Soc Transl. 1962;20(2):105-171.

16. Steklov AV. The problem of cooling of an heterogeneous rigid rod. Communs Kharkov Math Soc Ser. 1896;2(5):136-181.

17. de Rham G. Vari et es dierentiables. Herman, Paris; 1960.

18. Marcinkiewicz J. Sur les multiplicateurs des s eries de Fourier. Studia Mathematica. 1939;8(1):78-91.

19. Temam R. Behavior at $\mathrm{t}=0$ of the solutions of the semi-linear evoluation equations. J Di Equats. 1982;43(1):73-92. 